ARTICLE

Check for updates

https://doi.org/10.1038/s41467-021-24484-z

\title{
Single-molecule, full-length transcript isoform sequencing reveals disease-associated RNA isoforms in cardiomyocytes
}

\author{
Chenchen Zhu'1, ${ }^{12}$, Jingyan Wu ${ }^{1,12}$, Han Sun ${ }^{1}$, Francesca Briganti (1) 2,3,4, Benjamin Meder ${ }^{1,5,6,7}$ \\ Wu Wei(i] ${ }^{8,9,10 凶}$ \& Lars M. Steinmetz (iD) $1,2,3,10,11 \times$
}

\begin{abstract}
Alternative splicing generates differing RNA isoforms that govern phenotypic complexity of eukaryotes. Its malfunction underlies many diseases, including cancer and cardiovascular diseases. Comparative analysis of RNA isoforms at the genome-wide scale has been difficult. Here, we establish an experimental and computational pipeline that performs de novo transcript annotation and accurately quantifies transcript isoforms from cDNA sequences with a full-length isoform detection accuracy of $97.6 \%$. We generate a searchable, quantitative human transcriptome annotation with 31,025 known and 5,740 novel transcript isoforms (http://steinmetzlab.embl.de/iBrowser/). By analyzing the isoforms in the presence of RNA Binding Motif Protein 20 (RBM20) mutations associated with aggressive dilated cardiomyopathy (DCM), we identify 121 differentially expressed transcript isoforms in 107 cardiac genes. Our approach enables quantitative dissection of complex transcript architecture instead of mere identification of inclusion or exclusion of individual exons, as exemplified by the discovery of IMMT isoforms mis-spliced by RBM20 mutations. Thereby we achieve a path to direct differential expression testing independent of an existing annotation of transcript isoforms, providing more immediate biological interpretation and higher resolution transcriptome comparisons.
\end{abstract}

\footnotetext{
${ }^{1}$ Department of Genetics, School of Medicine, Stanford University, Stanford, USA. ${ }^{2}$ European Molecular Biology Laboratory (EMBL), Genome Biology Unit, Heidelberg, Germany. ${ }^{3}$ Cardiovascular Institute and Department of Medicine, Stanford University, Stanford, USA. ${ }^{4}$ Collaboration for joint PhD degree between EMBL and Heidelberg University, Faculty of Biosciences, Heidelberg, Germany. ${ }^{5}$ Institute for Cardiomyopathies Heidelberg (ICH), Heart Center Heidelberg, University of Heidelberg, Heidelberg, Germany. ${ }^{6}$ DZHK (German Center for Cardiovascular Research), partner site Heidelberg, Heidelberg, Germany. ${ }^{7}$ Department of Medicine III, University of Heidelberg, Heidelberg, Germany. ${ }^{8}$ CAS Key Laboratory of Computational Biology, Shanghai Institute of Nutrition and Health, University of Chinese Academy of Sciences, Chinese Academy of Sciences, Shanghai, China. ${ }^{9}$ Center for Biomedical Informatics, Shanghai Engineering Research Center for Big Data in Pediatric Precision Medicine, Shanghai Children's Hospital, Shanghai Jiao Tong University, Shanghai, China. ${ }^{10}$ Stanford Genome Technology Center, Stanford University, Palo Alto, USA. ${ }^{11}$ DZHK (German Center for Cardiovascular Research), partner site EMBL Heidelberg, Heidelberg, Germany. ${ }^{15}$ These authors contributed equally: Chenchen Zhu, Jingyan Wu. ${ }^{\bowtie}$ email: wuwei@picb.ac.cn; larsms@stanford.edu
} 
$\mathrm{N}$ early all human multi-exon genes are alternatively spliced $^{1-4}$, allowing a single gene to generate multiple RNA isoforms that give rise to different protein isoforms $s^{5,6}$, which consequently drive phenotypic complexity in eukaryotes ${ }^{1}$. Numerous diseases including cancers, neurological, and cardiovascular diseases, are associated with alternative splicing dysregulation, such as mutations in the heart-specific alternative splicing regulator, $R B M 20$, that cause dilated cardiomyopathy (DCM $)^{7-9}$. The conventional method to study alternative splicing is short-read RNAseq $1,4,10-13$, which sequences fragments of RNA and can therefore only detect expression changes of the whole gene or individual exons. Although software tools such as Kallisto ${ }^{14}$ or Salmon ${ }^{15}$ permit transcript level quantification with short-read RNA-seq, this requires either a reference annotation or de novo transcriptome assembly. The latter, needed for discovery of novel isoforms, is particularly challenging from short-read data. Therefore, short-read RNA-seq is not ideal for quantitatively detecting individual RNA isoforms. The emergence of long-read sequencing technologies including Pacific Biosciences (PacBio) and Oxford Nanopore Technologies (ONT) have provided a new tool for more comprehensive analysis of alternative splicing. An initial, genome-wide study with PacBio used 476,000 reads ${ }^{16}$, which enables de novo transcript identification but is insufficient for accurate quantification. Consequently, subsequent studies with PacBio or ONT focused on quantifying selected genes using enrichment methods, lacking genome-wide profiles ${ }^{17-20}$. Recent studies have quantified transcripts using long-read sequencing in mouse ${ }^{21,22}$ and breast cancer cells ${ }^{23}$, however no quantitative differences between samples were assessed. Furthermore, long-read sequencing has just begun to be utilized in single-cell transcriptomics for studying transcriptional heterogeneity 24,25 . It is challenging to apply long-read RNA-seq to determine global expression changes of transcripts, because: (1) low sequencing depth often does not allow adequate genome coverage for accurate quantification; (2) high sequencing error rates require novel computational methods that allow transcript quantification, classification, and visualization; (3) high false discovery rates of identified unannotated transcripts due to sequencing artifacts such as undesired template switching of reverse transcriptase (RT) and off-target priming of oligo $(\mathrm{dT})^{21}$ confound downstream analysis. Thus, quantitative, genome-wide differential expression analysis to detect isoform changes at scale has not been possible so far. This has made it difficult to identify the molecular mechanisms underlying many diseases in which splicing has been implicated. Here, we demonstrate that single-molecule full-length RNA sequencing enables to identify disease-associated transcript isoforms.

\section{Results and discussion}

Generation of long-read RNA-seq dataset with ONT sequencing. We generated a large, long-read dataset for human induced pluripotent stem cell derived cardiomyocytes (iPSC-CMs) using ONT sequencing of cDNA and developed a workflow that accurately and quantitatively measures and compares full-length splicing isoforms at genome-scale independent of an existing annotation. RNAs from human iPSC-CMs (each with two independent clones) with and without DCM-associated RBM20 mutations, together with spike-in controls $\left(\mathrm{ERCC}^{26}\right.$ and sequins ${ }^{27}$ ), were converted to full-length cDNAs and sequenced with ONT MinION technology (Method section, Supplementary Fig. 1, and Supplementary Table 1). In total, we generated 21 million high quality reads (mean qscore $\geq 6$, Method section, Supplementary Fig. 2), and quantified 36,765 transcript isoforms for a total of 11,707 genes, covering $53.8 \%(10,682 / 19,847)$ of the protein-coding genes in the human genome (Fig. 1, Supplementary Fig. 3, and Supplementary Tables 1-3). In comparison, our complementary short-read data with $\sim 1200$ million pair-end reads identified 16,726 protein-coding genes (read count $>1$ ).

Development of FulQuant to define full-length transcriptomes. Major problems of current methods to quantitate isoforms using long-read sequencing are sequencing artifacts and high sequencing errors resulting in high false transcript discoveries. To address these challenges, we established a computational method for full-length transcript quantification (FulQuant), which integrates accurate full-length read identification, transcript quantification, and visualization from ONT cDNA sequences. This new method allows de novo transcript annotation and employs stringent and complex criteria for filtering reads, alignments, and transcripts, to rule out artifacts from RT and sequencing errors, and groups reads into transcripts based on their splice sites, generating a set of highly confident transcripts with abundance estimates (Methods section and Supplementary Fig. 4). To

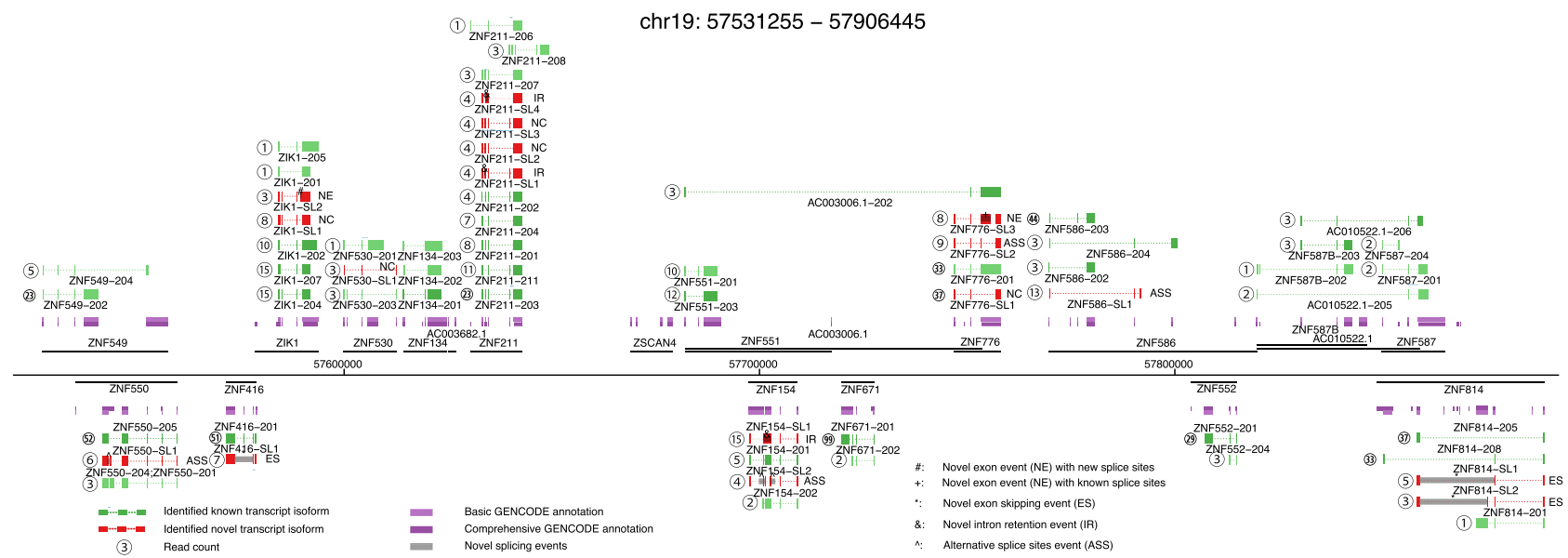

Fig. 1 Complex landscape of full-length human iPSC-CM transcriptome. Genome-wide measurement of full-length splicing isoforms in human iPSC-CMs with a region of chromosome 19 (chr19:57531255-57906445) as an example. Gene loci are presented as a horizontal black line with annotated genes on + or - strands above or below the genome axis, respectively. Collapsed GENCODE comprehensive and basic annotation are presented in dark purple and purple track. Known transcript isoforms are shown as green tracks, and previously unidentified transcript isoforms as red. Known transcript isoforms did not pass transcript filtering criteria are presented in light green. Read counts for each identified transcript are given as number in circle. Based on the novel splicing events (location indicated with gray box and text symbols), novel transcript isoforms are categorized as novel exon combination (NC), novel exon (NE), novel intron retention (IR), novel exon skipping (ES), or novel alternative splice sites (ASS). Dubious novel read through transcripts are not shown. 
a

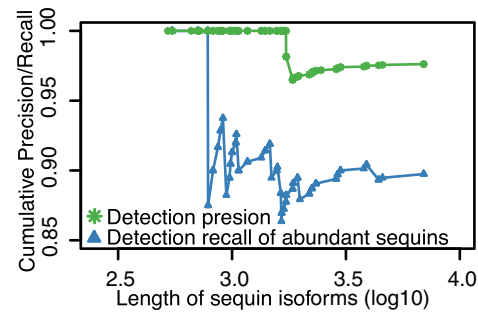

b
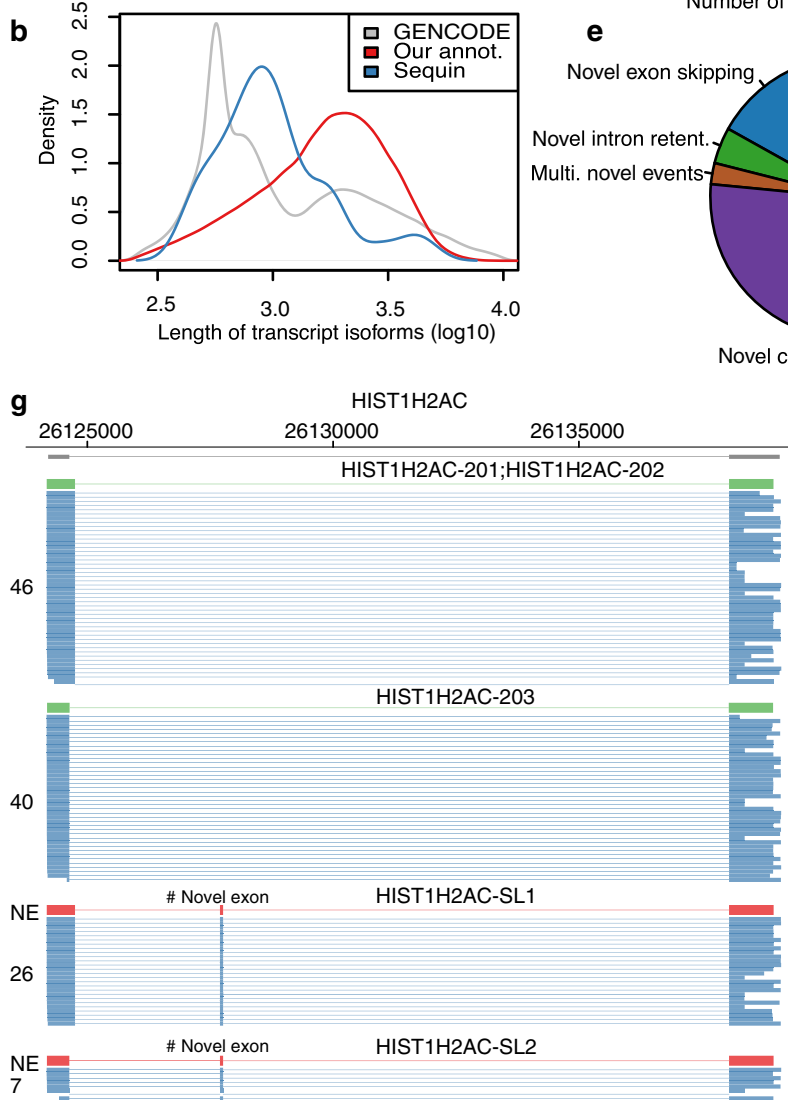

c

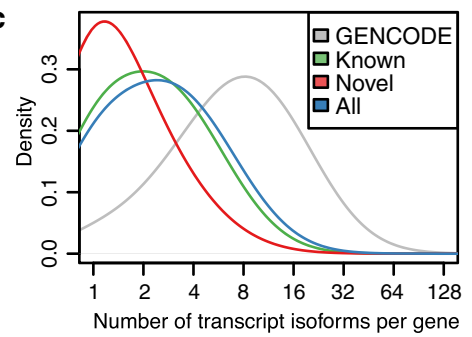

d
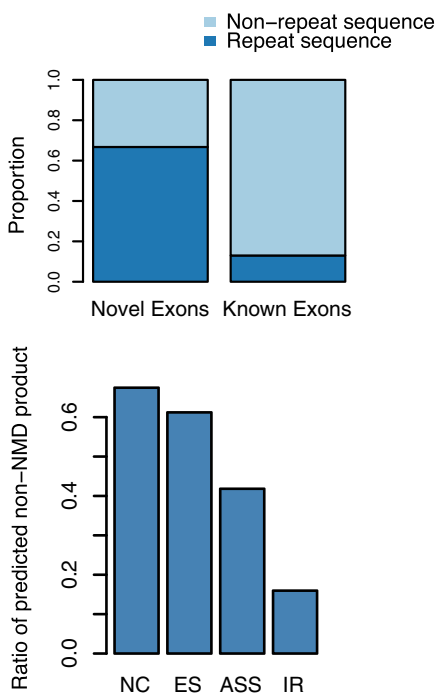

PI4KB

$151310000 \quad 151320000$

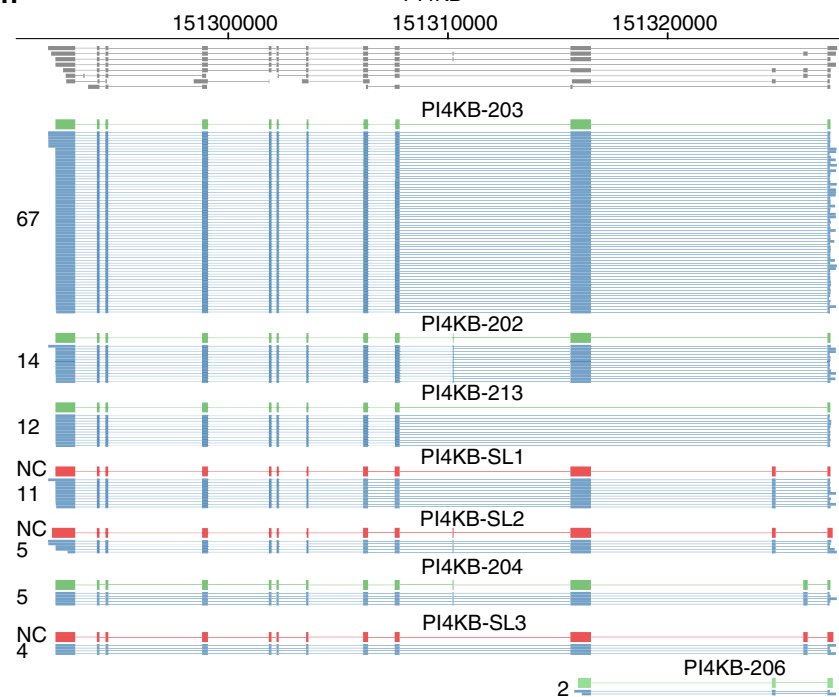

Fig. 2 FulQuant method accurately identifies transcript isoforms in iPSC-CMs. a Length dependent precision (green) and recall (blue) of the FulQuant method as estimated using all or the most abundant $50 \%$ synthetic sequin controls. $\mathbf{b}$ Length distribution of transcripts in GENCODE, of our identified isoforms, and of the sequin controls. $\mathbf{a}, \mathbf{b}$ are vertically aligned with the same $\mathrm{x}$ axis to illustrate the relevance of the accuracy estimated by sequins on human transcripts. c Distribution of number of isoforms per gene based on GENCODE comprehensive annotation and the transcripts identified in this study (divided into Known, Novel, and All). d Proportion of known exons that contain repeat sequences compared to the proportion of identified novel exons. e Percentages of novel transcripts isoforms by the types of novel splicing events: novel exon (NE), novel intron retention (IR), novel alternative splice sites (ASS), novel exon skipping (ES), and unannotated combinations of known exons (NC). Ambiguous transcripts are those with alignment issues. $\mathbf{f}$ Ratio of predicted non-NMD product in each novel transcript categories. $\mathbf{g}$, $\mathbf{h}$ Examples of genes with unannotated combinations of known exons and novel exon, respectively. Known isoforms in the comprehensive GENCODE annotation are shown in gray track. Isoforms with $<5$ reads are not shown. Source data are provided as a Source Data file.

evaluate FulQuant's accuracy to identify isoforms, we determined its performance on synthetic sequencing spike-in sequences (sequins, spiked in at $3 \%$ of total RNA) spanning a $\sim 10^{6}$-fold range in concentration that covers the dynamic range of gene expression observed across the human transcriptome ${ }^{27}$. These spike-ins represent 72 artificial gene loci with multiple exons encoding 156 alternative isoforms to mimic human splice isoforms of different sequences, structures, abundances, and lengths. We identified 82 of the 156 sequin isoforms, including 90\% (70) of the $50 \%$ most concentrated isoforms, which account for 99.95\% of all sequin RNA molecules. Our true positive rate for isoform identification, defined as the ratio of identified reference transcripts over all reported transcripts, was $97.6 \%$ based on all sequin isoforms, with the largest sequin isoform being $\sim 7 \mathrm{~kb}$
(Fig. 2a). In all, 98.1\% of all spliced transcripts in human GENCODE annotation are below this $7 \mathrm{~kb}$ threshold (Fig. 2b). The true positive rate was $100 \%$ for isoforms shorter than $1728 \mathrm{bp}$, which is greater in length than $70 \%$ of all human spliced transcripts. The identification accuracy of FulQuant was higher than the existing long-read pipeline FLAIR $^{28}$, which yielded a $30 \%$ true positive rate when benchmarked against our sequin dataset using default settings (Supplementary Text). FulQuant's improvement in transcript identification over FLAIR comes primarily from removal of false positive transcripts resulting from artefacts in RT and sequencing that manifested in the synthetic controls. With FulQuant, the quantification of sequins correlated linearly with input concentrations (average Pearson correlation coefficient 0.85 , Supplementary Fig. 5). Furthermore, $98.8 \%$ of transcript 
boundaries were positioned within $20 \mathrm{bp}$ of the annotated transcripts (Supplementary Fig. 6), indicating a reliable capture of full-length transcripts without relying on annotation. Likewise, when we applied FulQuant to our human iPSC-CM dataset, we observed a high correlation between technical and biological replicates (Supplementary Fig. 7), and accurate boundary definition of full-length transcripts (Supplementary Tables 2 and 3). FulQuant's quantification generated better agreement between biological replicates than FLAIR (Supplementary Fig. 7), likely due to reduced number of false positive transcripts.

Global analysis of full-length transcriptomes in human iPSCCMs. Our data revealed genome-wide complex alternative splicing patterns for known and novel transcript isoforms. By comparing our transcript isoforms to the human GENCODE annotation (V24), we identified 36,765 transcript isoforms, 5740 (15.6\%) of which were novel. For example, we identified eight known and four novel transcripts for the zinc-finger gene, ZNF211(Fig. 1). When comparing to a comprehensive annotation set CHESS (v2.2) ${ }^{29}, 3028$ (8.2\%) were novel (Supplementary Text and Fig. 8). Our genome-wide full-length isoform dataset is available via a searchable browser, which we have developed and made freely available (http://steinmetzlab.embl.de/iBrowser/).

Our identified transcript isoforms have lengths from $177 \mathrm{bp}$ to 10,718 bp with a median of 1768 bp (Fig. $2 b$ and Supplementary Fig. 9). The length distribution is similar to GENCODE full-length protein-coding and lincRNA transcripts (Supplementary Fig. 10), however we did not capture large transcripts such as TTN isoforms ( $100 \mathrm{~kb}$ in size, a major structure component of the heart muscle) likely due to RT limitation of cDNA synthesis. A median of two alternatively spliced transcript isoforms per gene were identified (Fig. 2c). It is notable that by applying our approach to only one cell type, namely iPSC-CMs, we identified already $24.1 \%$ of proteincoding isoforms $(n=80,901)$ covering 9946 genes in the GENCODE comprehensive annotation, which cumulates data from various human cell lines, tissues, and population. This reflects the comprehensive nature and depth of our long-read approach, and suggests that the number and complexity of all splice isoforms in the human genome are likely underestimated.

Aberrant splicing may generate novel transcripts, which could be used for diagnostics and drug development. Notably novel exons detected in our dataset were enriched in repetitive sequences defined by RepeatMasker (Fig. 2d), with $21 \%$ of novel exons overlapping SINE/Alu sequences. In addition to a higher degree of intron retention (IR, 4.1\%), most novel transcript isoforms contained alternative splice sites (ASS, 29.2\%), novel exon skipping (ES, 12.7\%), novel exons (NE, 8.6\%), and unannotated combinations of known exons (NC, 42.9\%, Fig. 2e, g, h). To assess the performance of our pipeline, we have successfully validated 26 out of 33 novel splice events (exon skipping, intron retention, and novel exons) using independent fragment analysis by RT-PCR (Methods section, Supplementary Figs. 11 and 12 and Supplementary Table 6). In terms of protein-coding potential, our identified novel transcript isoforms add 1 potential protein-coding isoform every four genes on average (Fig. 2f and Supplementary text). Supporting the resolution of our data, we also identified 45 potential spliced polycistronic transcripts from adjacent genes (Supplementary Fig. 13) and unspliced polycistronic transcripts that are transcribed

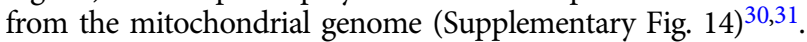

Analysis of coordinated exon usage events in human iPSCCMs. Coordinated expression of exons underlies many regulatory mechanisms ${ }^{23}$. Its analysis also provides important insights into strategies of alternative splicing ${ }^{32,33}$. Particularly in heart, mutually exclusive exon pairs in genes such as TPM1, 2 and 3 have been used as model systems to study splicing regulation ${ }^{34}$. Our long-read data enabled a first systematic analysis of the interdependency between adjacent and distant exons across an entire transcript isoform in heart cells. We identified 2793 significantly co-regulated exon pairs in 722 genes (adjusted $P<$ 0.001 , mutually exclusive and inclusive association, Methods section and Supplementary Table 4). For example, we detected the well-documented exon pair association in the heart-specific gene, TPM1, that harbors adjacent mutually exclusive exons 2 and 3 , which are representative of muscle type switching between smooth and skeletal muscle ${ }^{35}$. In addition, we identified 6 further co-association events in TPM1 transcript isoforms, including 4 distal pairs, such as a mutually inclusive pair between exons 2 and 11 and a mutually exclusive pair between exons 7 and 11 (Fig. 3a). Another prominent example is MYL7 (Fig. 3b), a critical gene for cardiac function, that contains 18 mutually inclusive (6) and exclusive (12) exon pairs. Over $70 \%$ of all tested exon pairs (25) in this gene were significantly co-associated, and the majority (61\%) are distally positioned, suggesting complex splicing regulation for this gene. Overall, we observed that adjacent co-association, within one or two exons, is more common than distal coassociation (Supplementary Fig. 15). Since splicing of nascent RNAs occurs co-transcriptionally at an extremely fast rate (" $50 \%$ of splicing is complete within $\sim 1.4 \mathrm{~s}$ after $3^{\prime}$ splice site synthesis") ${ }^{36}$, we reasoned that this theoretically favors prompt correlated splicing of adjacent, newly synthesized exons.

Identifying mis-spliced full-length transcript isoforms in RBM20 mutants. Aberrant splicing is linked to a wide variety of diseases, however identifying the causative event is challenging. Our approach using full-length transcript sequencing provides an opportunity to study differential isoform expression between different states, which will enable the direct comparison between heath and disease. To develop a quantitative approach to identify differentially expressed transcript isoforms between sample types, we applied our pipeline to studying how full-length splicing isoforms are affected by mutations in the heart-specific splicing regulator $R B M 20^{37-39}$. RMB20 mutations (such as R634Q within its arginine/serine-rich domain) lead to an aggressive form of DCM and short-read sequencing has shown that RBM20 target exons are mis-spliced in rat $^{37}$, mouse ${ }^{40}$, pig $^{41}$, and human cardiac cells ${ }^{38,42}$. However, in many of these targets it has remained unclear to which aberrantly spliced transcript isoform these differentially spliced exons belong and whether that may or may not produce malfunctioning proteins, or may simply lead to the absence of correctly spliced protein. In addition to the $6 \mathrm{M}$ reads for the R634Q mutant, we also generated 9M reads for the P633L mutant, a new pathogenic mutation (proline-to-leucine change at amino acid position 633) that we recently identified ${ }^{42,43}$. We performed de novo transcript annotation using R634Q and wildtype data. While this approach would not identify novel isoforms seen only in the P633L mutant, it allowed us to quantitatively compare the expression level of isoforms detected in R634Q and wild type, across all mutants. To compare full-length transcriptomes of iPSC-CMs harboring mutants R634Q and P633L to wild-type $R B M 20$, we performed differential isoform expression analysis using DESeq $2^{44}$ on the quantified transcripts (Supplementary Table 3). We identified 121 transcript isoforms in 107 genes that are differentially expressed (adjusted $P<0.001$, Fig. 4a, Supplementary Table 5, and Methods section). Both mutants showed similar expression levels (correlation coefficient $=0.77$ ) and did not differ significantly when compared to each other using differential expression analysis (Supplementary Fig. 16). Over $80 \%$ of our candidates identified by long-read sequencing were recapitulated by the same analysis run on the short-read 


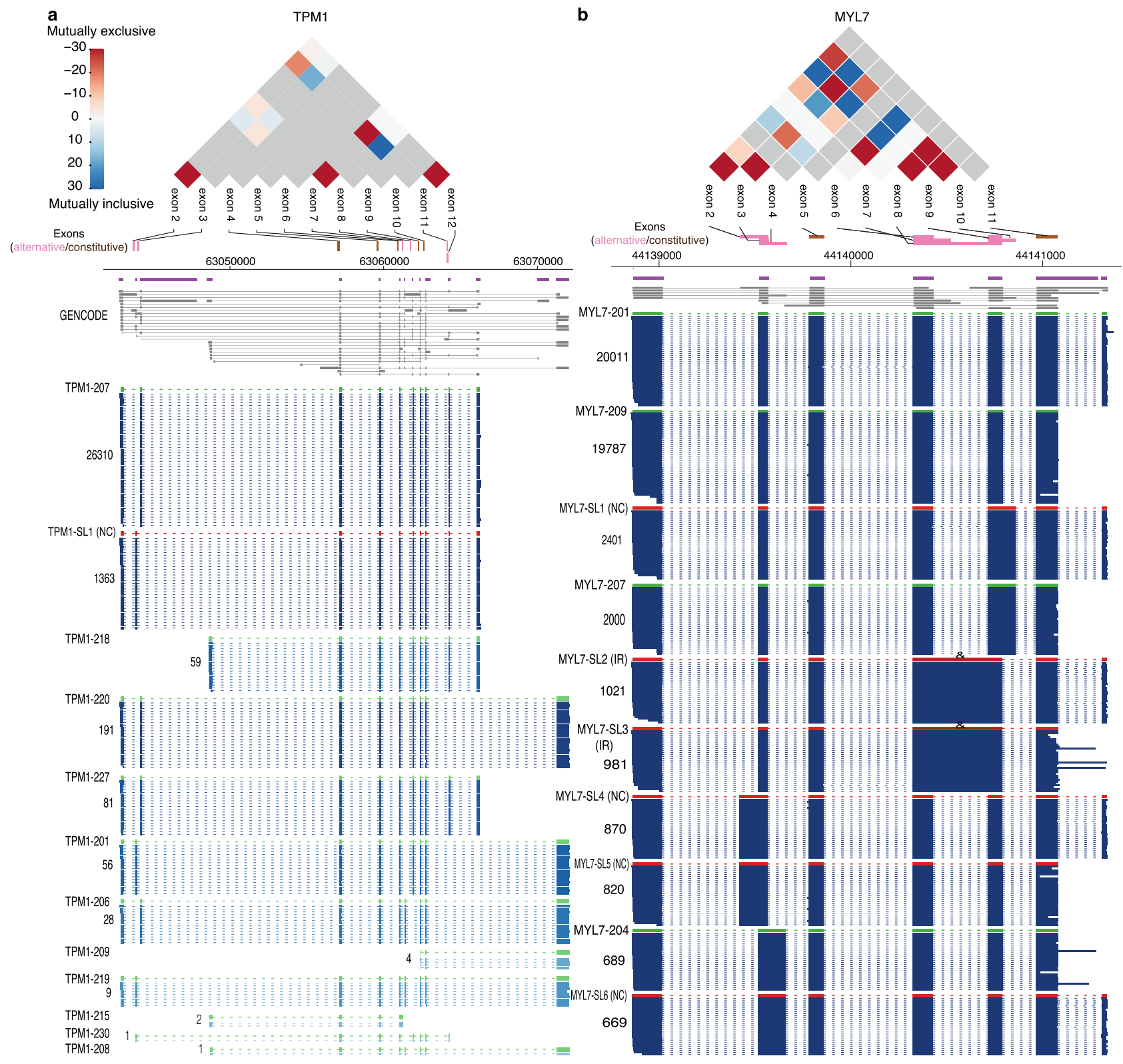

Fig. 3 Exon co-associated events in human iPSC-CMs. TPM1 (a) and $M L Y 7$ (b) are shown as examples of genes containing multiple adjacent and distal mutually inclusive or exclusive alternative exons. The co-association strength ( $\log 10$ of $p$-value) between alternative exon pairs is illustrated in the triangle plot for mutually exclusive (red, - ) and inclusive pairs (blue, + ), while the gray color indicates exon pairs not tested. Purple and gray annotations are for collapsed and expanded GENCODE comprehensive annotation, respectively. Reads in blue are in the same log-scale as Fig. 1 with darkness correlating with the read counts. Only exons used for our co-association analysis are shown. Lowly abundant transcript isoforms are not shown for illustration clarity. Source data are provided as a Source Data file.

data with transcript isoform quantification using Kallisto (Methods section, Supplementary text, and Supplementary Figs. 17 and 18). Gene ontology analysis of these 107 genes showed enrichment in cardiac functions, like striated muscle development, regulation of heart contraction, ion transport, and actin filament-based processes (adjusted $P<0.05$ ), supporting the essential role of $R B M 20$ in cardiac function (Supplementary text and Supplementary Fig. 19).

Notably, not all 107 genes with differentially expressed transcript isoforms had significant changes in total gene expression level, as assessed using standard differential gene expression analysis (Methods section and Fig. $4 \mathrm{~b}$ ). Among the 107 genes, 27 expressed a single isoform, and of the remaining 80 genes with multiple isoforms, 69 genes displayed total gene expression differences. Among these 69 genes, 60 genes showed differences in major isoform expression ( $>50 \%$ of total transcripts of gene), while 9 genes differentially expressed only minor isoforms. Eleven genes with multiple isoforms showed no change in total gene expression but differentially expressed one or more isoforms. When assessing transcript isoform usage, 8 out of these 11 genes showed significant difference in isoform ratios between mutant and wild-type (Fisher's exact test, adjusted $P$-value $<$ 0.005, Methods section, and Supplementary Fig. 20), clearly suggesting complex regulation at the level of splicing, independent of promoter or isoform stability differences since total gene expression was not affected (Supplementary Table 5). This complex isoform regulation demonstrates that measuring differential RNA expression at the gene level and not at the isoform 


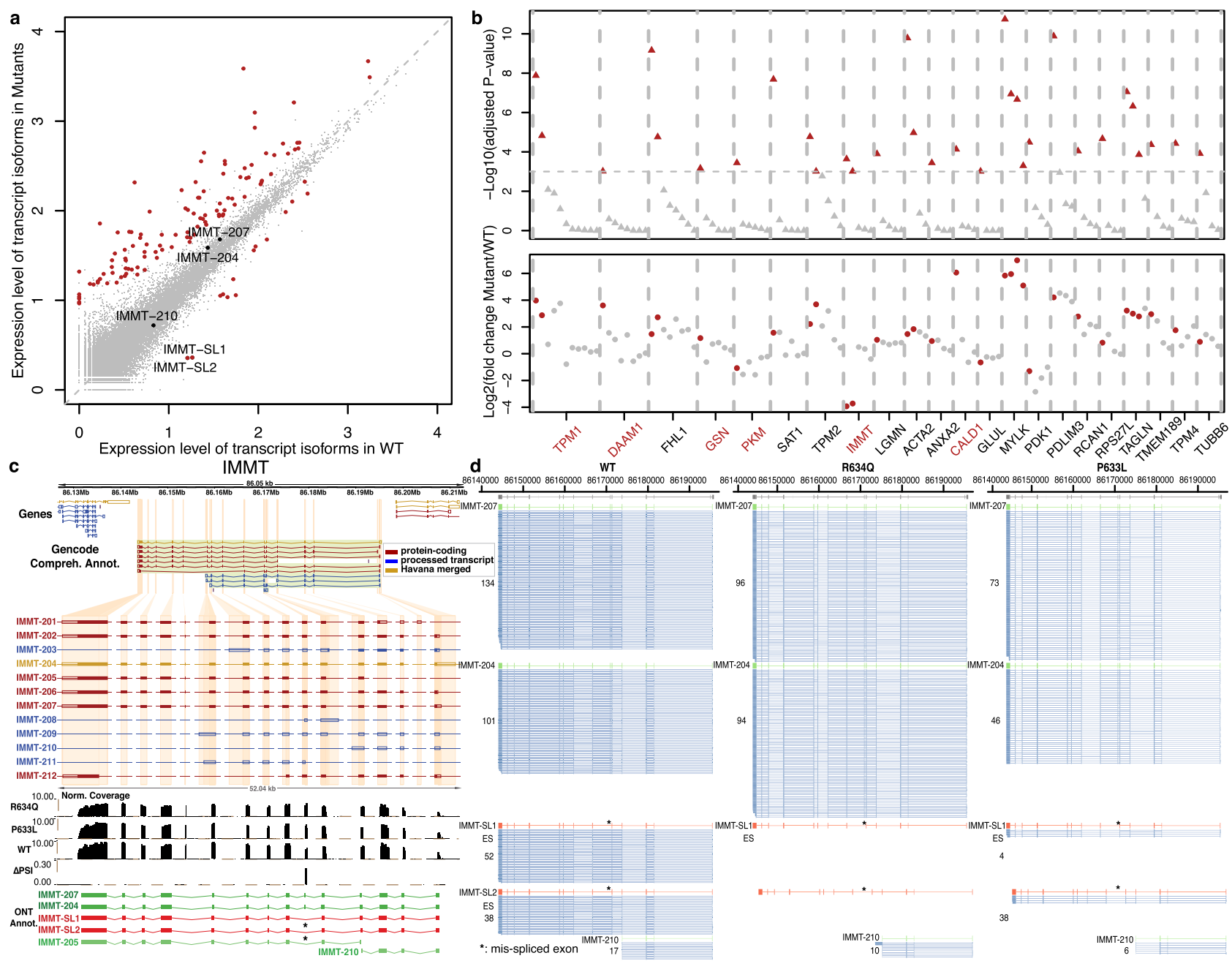

Fig. 4 Differentially expressed full-length transcript isoforms in the RBM20 mutants R643Q and P633L. a Scatter plot for average full-length isoform expression levels in WT and mutants. Each dot represents an isoform, with red dots highlighting the differentially expressed ones (adjusted $P<0.001$ ). Isoforms for IMMT gene are labeled. b Genes containing differentially expressed isoforms. Top, significance level of the differential expression analysis for each isoform (triangle) with significant ones in red. Bottom, fold change of expression level of each isoform (point) with significant ones in red. Gene names in red are genes differentially expressed only proportion of their isoforms, with no significant change at the total gene expression level. Only genes with more than three expressed isoforms are displayed. c IMMT gene with GENCODE annotation, short-read data and identified transcript isoforms in longread data of this study. Tracks from top to bottom: collapsed view of GENCODE transcripts around IMMT locus, expanded view of IMMT GENCODE transcripts, short-read coverage for mutant and WT, delta-PSI between mutant and WT inferred in short-read data, and known (green) and novel (red) transcripts identified in long-read data. GENCODE transcript type are color-coded with red (protein-coding), blue (processed) and gold (Havana-merged). Coding regions are highlighted by filled color in the GENCODE annotation tracks. d Side-by-side comparison of IMMT isoforms expression in RBM2O R634Q, P633L mutant and WT. Two novel transcript isoforms in red, IMMT-SL1 and IMMT-SL2, were only expressed in WT samples. Known GENCODE transcripts are in green and raw long-reads are in blue. The mis-spliced exon 6 is indicted by asterisk. IMMT-205 and IMMT-208 were omitted due to low read count ( 1 in all samples). Source data are provided as a Source Data file.

level can fail to detect significant expression changes in specific isoforms, and approaches that do not require prior annotation are advantageous. Two prominent examples of genes with differential expression of specific transcript isoforms (and not total gene expression) are $T P M 1^{37}$ and $I M M T^{38}$, both previously reported to be mis-spliced from short-read sequencing of rats and/or human cardiac transcriptomes. Our long-read data showed clearly that not all isoforms are equally affected in the mutants (Fig. 4b). For IMMT, our percentage of exon inclusion (PSI) analysis on short-read data identified only exon 6 as differentially spliced between wild-type and the mutants, as reported in previous studies $37,38,41,42$ (Fig. 4c and Supplementary Text). However, the effect of mis-splicing on full-length transcript isoforms was not captured by previous short-read analyses ${ }^{37,38}$.
Specifically, it remained unclear how many and which transcripts are affected by RBM20 mutations. For IMMT, based on the reference GENCODE annotation, it is logical to hypothesize transcript IMMT-205 as the only affected isoform, as this is the only annotated transcript of IMMT without exon 6. Differential expression analysis on transcript quantification based on short-read data indeed reported IMMT-205 as the only significant hit for IMMT (Methods section). This is misleading because our long-read based approach could clearly identify two novel IMMT transcript isoforms, IMMT-SL1 and IMMT-SL2, that lack exon 6 compared to their corresponding known transcripts, IMMT-207 and IMMT-204. These two isoforms are expressed in the wild type, absent in R634Q and only marginally expressed in P633L, in contrast to three isoforms that are not differentially expressed (Fig. 4a, d). Only one 
read was identified for IMMT-205 in all samples. Based on domain analysis, we found that the lack of exon 6 may result in proteins with new functions, as the absence of 32 amino acids encoded by this exon disrupts one existing coiled-coil domain and an intrinsically disordered region in the protein (Supplementary Fig. 21). Thus, the discovery of novel IMMT isoforms illustrates the utility of full-length transcriptome identification and quantification without prior annotation.

\section{Discussion}

In conclusion, we present a genome-wide analysis of full-length transcript isoforms of human cells and its differential analysis in the context of splicing mutations in a critical heart gene, RBM20. While differential exon usage has been described in splicing-deficient RBM20 mutations $37,38,40-42$, we discovered complex isoform deregulation on select transcripts, that likely reflects complex combinations of promoter activity, isoform stability and alternative splicing changes on individual genes in RBM20 mutants. Our FulQuant workflow allows direct identification and quantification of transcript isoforms without prior annotation. Exemplified by two novel IMMT transcript isoforms that are alternatively spliced, annotation-free full-length isoform sequencing is critical for identifying the existence of many new, informative transcript isoforms that were previously missed. These observations, combined with the overall, intricate complexity of the human transcript isoform landscape (depicted in Fig. 1 and in the genome browser), demonstrate the critical need for more widespread adoption of long-read sequencing for transcriptome studies to provide context to previously identified alternatively spliced exons. Knowing the full-length isoform is essential to understanding the functional product of an expressed gene. Our workflow to quantify and compare full-length transcript isoforms directly, including various classes of novel transcripts, can be readily extended to other cell types and tissues, and the many diseases in which aberrant splicing has been proposed to play a role.

\section{Methods}

Genome editing in human iPSCs. Methods for genome editing and iPSC-CM generation are detailed in our publication ${ }^{42}$. Briefly, we obtained human iPSCs from the Stanford Cardiovascular Institute Biobank. Annealed (T4 ligation buffer, NEB) and phosphorylated (T4 PNK, NEB) guide RNA (gRNA, designed using tools at http://crispr.mit.edu) oligonucleotides were introduced into the BbsI sites of the pSpCas9(BB)-2A-GFP plasmid and transformed into STBL3 E. coli cells and verified via Sanger sequencing. One day after plating the cells into Matrigel coated 6-well plates at low density in Essential 8 (E8) media, media was changed to E8 with Rock inhibitor (Tocris Cat. No. 1254). For each well, CRISPR/Cas9 vector ( $1 \mu, \mathrm{pSpCas} 9(\mathrm{BB})-2 \mathrm{~A}-\mathrm{GFP})$ and $4 \mu \mathrm{g}$ of single-stranded DNA donor (Supplementary Information) were introduced into the cells via transfection with Lipofectamine 3000. GFP + cells were isolated $36-48 \mathrm{~h}$ after transfection using a FACSAria IIu (DB Biosciences) flow cytometer with a $100-\mu$ m nozzle, maintained in E8 with Rock inhibitor for the first 3 days with an initial density of $2-3 \times 10^{3}$ cells/well, and subsequently transferred to regular E8 until the colonies size reached $\sim 0.5 \mathrm{~mm}$. Individual iPSC clones were isolated and re-plated in a well of a 24-well plate in E8 with Rock inhibitor. The homozygous edits were confirmed by sequencing after PCR amplification of the target genomic region (Supplementary Information). The R634Q cells will be made available upon request.

iPSC-CM generation. iPSCs were differentiated into cardiomyocytes as a monolayer through the modulation of WNT signaling. iPSCs were plated at low density on Matrigel coated plates to have them $70-80 \%$ confluent after 4 days (Day 1 of differentiation). Differentiation was induced with $3 \mathrm{ml}$ of RPMI 1640 (Life Technologies 11875-093) with $1 \mathrm{X} \mathrm{B} 27^{\circledR}$ Minus insulin (Life Technologies 0050129SA), supplemented with $6 \mu \mathrm{M}$ CHIR (TOCRIS 4953). On day 6 , media was replaced with $3 \mathrm{ml}$ of RPMI 1640 with $1 \mathrm{X} \mathrm{B27}{ }^{\circledR}$ Minus Insulin, supplemented with $5 \mu \mathrm{M}$ IWR. From day 8, cells were kept in RPMI 1640 (Life Technologies 11875-093) with 1X B27 ${ }^{\circledR}$ Serum-Free Supplement (Life Technologies 17504-044). From days 12-15 cells were treated with RPMI 1640 no Glucose (Life Technologies 11879020 ) with 1 X B27, then allowed to recover for 2 days in RPMI 1640 with 1X B27, and subsequently replanted at a density of $3 \mathrm{Mi}$ cells/well. Cells were then maintained in RPMI 1640 with 1X B27 for 4 more weeks before being collected for the experiment.
Reverse transcription. RNAs were cleaned and treated with DNase using ZymoResearch RNA Clean and Concentrator-5. A total of $4.5 \mu \mathrm{L}$ mixture containing $5 \mathrm{ng}$ of purified iPSC-CM RNAs with $2.8 \%$ ERCC and 3\% Sequins Version A, $1 \mu \mathrm{L}$ of $10 \mu \mathrm{M}$ oligodT (/5SpC3/A*A*G*CAGTGGTATCAACGCAGAGTACTTTTTTTTTTTTTT TTTTTTTTTTTTTTTTVN), $1 \mu \mathrm{L}$ of $10 \mathrm{mM}$ dNTP, $0.1 \mu \mathrm{L}$ of Takara recombinant ribonuclease inhibitor $(40 \mathrm{U} / \mu \mathrm{L})$ were incubated at $72{ }^{\circ} \mathrm{C}$ for $3 \mathrm{~min}, 4^{\circ} \mathrm{C}$ for $10 \mathrm{~min}$, and $25^{\circ} \mathrm{C}$ for $1 \mathrm{~min}$. Another $5.5 \mu \mathrm{L}$ mixture containing $2 \mu \mathrm{L}$ of $5 \mathrm{x}$ SuperScript II buffer, 2 $\mu \mathrm{L}$ of $5 \mathrm{M}$ betaine, $0.5 \mu \mathrm{L}$ of $100 \mathrm{mM}$ DTT, $0.5 \mu \mathrm{L}$ of SuperScript II $(200 \mathrm{U} / \mu \mathrm{L}), 0.25 \mu \mathrm{L}$ of Takara recombinant ribonuclease inhibitor, $0.1 \mu \mathrm{L}$ of $100 \mu \mathrm{M}$ TSO, $0.09 \mu \mathrm{L}$ of nuclease-free water, and $0.06 \mu \mathrm{L}$ of $1 \mathrm{M} \mathrm{MgCl}_{2}$ were added to obtain a final reaction volume of $10 \mu \mathrm{L}$. The final $10 \mu \mathrm{L}$ RT reaction were incubated at $42^{\circ} \mathrm{C}$ for $90 \mathrm{~min}, 10$ cycles of $50^{\circ} \mathrm{C}$ for $2 \mathrm{~min}$, and $42^{\circ} \mathrm{C}$ for $2 \mathrm{~min}^{4}$. The reaction was then stopped by incubation at $70^{\circ} \mathrm{C}$ for $15 \mathrm{~min}$ and held at $4{ }^{\circ} \mathrm{C}$. The reaction was then treated with $1 \mu \mathrm{L}$ of $1: 10$ dilution of NEB Recjf (Catalog \# M0264S) at $37^{\circ} \mathrm{C}$ for $30 \mathrm{~min}$ and $65^{\circ} \mathrm{C}$ for $20 \mathrm{~min}$.

PCR amplification of full-length cDNA. $25 \mu \mathrm{L}$ of $2 x$ NEB Q5 HotStart master mix $0.25 \mu \mathrm{L}$ of $10 \mu \mathrm{M}$ PCR primers (ONT_Index1_ISPCR and ONT_Index2_ISPCR) ${ }^{5}$ and $14.5 \mu \mathrm{L}$ of nuclease-free water were added to the RT reaction. The PCR were performed as follows: $98^{\circ} \mathrm{C}$ for $30 \mathrm{~s}, 20$ cycles of $98^{\circ} \mathrm{C}$ for $10 \mathrm{~s}, 67^{\circ} \mathrm{C}$ for $15 \mathrm{~s}$, and $72{ }^{\circ} \mathrm{C}$ for $6 \mathrm{~min}$, then $72^{\circ} \mathrm{C}$ for $2 \mathrm{~min}$, and hold at $4^{\circ} \mathrm{C}$. PCR reactions were purified using 0.75 volume of Ampure XP beads and eluted with $25 \mu \mathrm{L}$ of nuclease-free water. Full-length cDNA were analyzed on Agilent Bioanalyzer using High Sensitivity DNA chips.

Oxford nanopore sequencing. In total, $1 \mu \mathrm{g}$ of cDNA were ligated with ONT sequencing adapter as described by the manufacturer with modifications in the following steps: (1) End repair and dA tailing; (2) Ligation of ONT sequencing adapter; (3) Addition of sequencing tether. Sequencing was performed using either R9.4 and R9.5 flow cells on the MinION device. Data were recorded with ONT's MinKNOW (1.4.2) software for $48 \mathrm{~h}$. Basecalling was performed using ONT's Albacore software (version 2.1.3) with the options '-disable_filtering -kit SQKLSK108‘ and corresponding flow cell version (Supplementary Table 1).

FulQuant for full-length transcript isoform quantification. Analysis was conducted in R (3.5.3) or using custom Bash scripts unless otherwise specified. We assessed the dependency of percentage of alignment identify (PID) on mean read quality (mean_qscore defined by ONT) and determined the mean read quality score cutoff 6 where over $80 \%$ reads have a PID $>80 \%$ (Supplementary Fig. 2). After removing low quality reads, we trimmed sequencing adapters ISPCR (AAGCAGTGGTATCAACGCAGAGTAC) and polyA sequences ( $\geq 10$ consecutive $\mathrm{A} / \mathrm{T}$ with one mismatched allowed) at both ends ( $200 \mathrm{bp}$ window) of the reads. Reads having adapter sequence at one end and polyA sequence at the other end were considered full-length. We aligned the reads against the human genome (GRCh38) using minimap2 (version: 2.9-r751-dirty) with the parameters: '-K500m -secondary $=$ no $-\mathrm{a}-\mathrm{x}$ splice - splice-flank $=$ yes $^{\prime}$. Alignments meeting the following criteria were removed from downstream analysis: (i) consist of supplementary alignments, (ii) size of soft clipping at either ends greater than $30 \mathrm{bp}$, (iii) insertion size near splice junctions (identified by cigar N) $>5$ bp, and (iv) unspliced alignment (singleton).

The transcriptome annotation was performed on data for WT and R634Q. We identified tag splice sites for intron-exon and exon-intron junctions using a clustering method previously described ${ }^{45}$ with the parameters 'HFWINSIZE $=5$ DISTHRES $=8$ PTHRESHOLD $=3$ SUMHOLD $=5^{\prime}$. Reads sharing the same tag splice sites were collapsed into consensus transcript isoforms. We include the terminal exons but do not consider transcript variation at $5^{\prime}$ and $3^{\prime}$ ends due to high error rate at both ends of the read. In fact, for the terminal exons, we annotated their splicing sites precisely, but not their transcript start (TSS) and stop (TES) sites. We used the median $5^{\prime}$ and $3^{\prime}$ positions of all reads in one transcript isoform as its TSS and TES sites. The following rules were used to remove false positive transcripts: (1) read count $>3$, (2) $>40 \%$ full-length reads, (3) transcript termination site $>10 \mathrm{bp}$ from known genomic polyA sequence, (4) strand information is available. Furthermore, we required in each gene locus that all transcripts: (1) had more than $3 \%$ read count of the most abundant transcript, (2) were not results of misalignment, and (3) were not $5^{\prime}$ truncation products of any identified transcripts with higher counts, unless the count is higher than $25 \%$ of the longer transcript. The code used to perform the above steps are attached in a supplementary file with detailed annotation. For the final annotation, we also removed transcripts that either had only 2 exons or may be a truncation product of a known transcript.

We compared our annotation with GENCODE human comprehensive annotation (V24) to classify transcripts as known and novel. Filtered transcripts mapping to known GENCODE annotation were rescued. We further annotated the novel transcripts into novel exons, novel combination, exon skipping, intron retention, alternative splice sites, and multiple events. A detailed definition of each novel transcript class can be found in the Supplementary Information and code.

Exon connectivity analysis. We first defined exons that were present in all transcripts as constitutive. For all exons pairs within a gene, we calculated the number of reads for when: (1) both exons are present, (2) only exon 1 is present, (3) only exon 2 is present, and (4) both exons are not present. Using this count 
matrix, we tested their co-association using fisher's exact test following $p$-values adjustment with $\mathrm{BH}$ method. Distal pairs were defined as exon pairs that were at least one constitutive exon apart.

Predicating nonsense-mediated decay events. For all novel transcripts that include a known start codon defined in the GENCODE annotation, we calculated the distance between the first stop codon and the last splice junction on the transcript. We defined transcripts as NMD products if this distance was $>55 \mathrm{bp}^{46}$.

Short-read data analysis. After mapping reads to GRCh38 using STAR v2.5.1b, gene expression levels were determined with featureCounts v1.6.0. For each exon, we counted inclusive and exclusive reads as reads that include the exon of interest and reads that include both the upstream and the downstream exon but not the exon of interest, respectively. Percentage of spliced-In (PSI) representing the ratio between inclusive reads over the sum of inclusive and exclusive reads was calculated. Transcript quantification was performed using Kallisto (0.46.2) with either GENCODE or our annotation as input annotation for index.

Gene and transcript isoform level differential expression analysis. Transcript quantification was achieved by counting number of reads sharing the same splice sites of that transcript. Gene quantification was achieved by summing transcripts counts in that gene locus. We compared CRISPR generated R643Q and P633L mutant samples to samples without CRISPR editing treatment (WT_NC) and non-edited samples with CRISPR editing treatment (WT), whereby standard differential expression analysis was performed using DESeq2 (1.26.0) for both genes and transcript isoforms. Due to the limited sequencing depth, we only considered genes and transcripts isoforms with more than 10 read counts in total in all samples for the test for the longread data. Significant candidates were defined using a $\mathrm{BH}$-adjusted $p$-value cutoff of 0.001 for the comparison between mutants and WT. For short-read data, same testing procedure with DESeq2 was applied to transcript and gene quantification obtained using Kallisto without any filtering. GO enrichment analysis was performed using PANTHER via the web interface at http://geneontology.org with the expressed genes as the reference list to control for background. To assess difference in transcript usage between mutant and wild type, we summarized biological replicates and performed Fisher's exact test for the 80 candidate genes with one or more differentially expressed isoforms. $P$-values were adjusted using $\mathrm{BH}$ procedure.

Validation of novel splicing events using fragment analysis. We used fragment analysis to validate 33 novel splicing events (Supplementary Table 6 and Supplementary Fig. 10). Oligo primers targeting the novel splicing events were designed such that amplicons with and without the splicing events would display detectable size differences on the gel. All primer sequences can be found in Supplementary Table 6. We performed standard RT-PCR and analyzed the fragment sizes using standard Bioanalyzer profiling. Only profiles contained band(s) with intended size(s) were considered as positive validation. For 7 unsuccessful cases, 3 cases didn't yield any PCR product and 4 cases contained fragments, which were at least $20 \mathrm{bp}$ off the predicted sizes.

Reporting summary. Further information on research design is available in the Nature Research Reporting Summary linked to this article.

\section{Data availability}

The long-read data generated in this study have been deposited in ArrayExpress under accession number E-MTAB-7334. The short-read data used in this study have been deposited in Sequence Read Archive (SRA) under accession number PRJNA579336 ${ }^{42}$. Our genome-wide full-length isoform dataset is available as a custom genome browser at http://steinmetzlab.embl.de/iBrowser/. Source data are provided with this paper.

\section{Code availability}

$\mathrm{R}$ code to perform the essential steps of transcripts filtering is available on GitHub https://github.com/czhu/FulQuant (doi: 10.5281/zenodo.4960275).

Received: 22 March 2021; Accepted: 22 June 2021;

Published online: 09 July 2021

\section{References}

1. Wang, E. T. et al. Alternative isoform regulation in human tissue transcriptomes. Nature 456, 470-476 (2008).

2. Pan, Q., Shai, O., Lee, L. J., Frey, B. J. \& Blencowe, B. J. Deep surveying of alternative splicing complexity in the human transcriptome by highthroughput sequencing. Nat. Genet 40, 1413-1415 (2008).

3. Brown, J. B. et al. Diversity and dynamics of the Drosophila transcriptome. Nature 512, 393-399 (2014).
4. Djebali, S. et al. Landscape of transcription in human cells. Nature 489, 101-108 (2012).

5. Lander, E. S. et al. Initial sequencing and analysis of the human genome. Nature 409, 860-921 (2001).

6. Johnson, J. M. et al. Genome-wide survey of human alternative pre-mRNA splicing with exon junction microarrays. Science 302, 2141-2144 (2003).

7. Scotti, M. M. \& Swanson, M. S. RNA mis-splicing in disease. Nat. Rev. Genet. 17, 19-32 (2016).

8. van den Hoogenhof, M. M., Pinto, Y. M. \& Creemers, E. E. RNA splicing: regulation and dysregulation in the heart. Circ. Res. 118, 454-468 (2016).

9. Lara-Pezzi, E., Gomez-Salinero, J., Gatto, A. \& Garcia-Pavia, P. The alternative heart: impact of alternative splicing in heart disease. J. Cardiovasc. Transl. Res. 6, 945-955 (2013).

10. Nagalakshmi, U. et al. The transcriptional landscape of the yeast genome defined by RNA sequencing. Science 320, 1344-1349 (2008).

11. Sultan, M. et al. A global view of gene activity and alternative splicing by deep sequencing of the human transcriptome. Science 321, 956-960 (2008).

12. Wilhelm, B. T. et al. Dynamic repertoire of a eukaryotic transcriptome surveyed at single-nucleotide resolution. Nature 453, 1239-1243 (2008).

13. Tilgner, $H$. et al. Comprehensive transcriptome analysis using synthetic longread sequencing reveals molecular co-association of distant splicing events. Nat. Biotechnol. 33, 736-742 (2015)

14. Bray, N. L., Pimentel, H., Melsted, P. \& Pachter, L. Near-optimal probabilistic RNA-seq quantification. Nat. Biotechnol. 34, 525-527 (2016).

15. Patro, R., Duggal, G., Love, M. I., Irizarry, R. A. \& Kingsford, C. Salmon provides fast and bias-aware quantification of transcript expression. Nat. Methods 14, 417-419 (2017).

16. Sharon, D., Tilgner, H., Grubert, F. \& Snyder, M. A single-molecule long-read survey of the human transcriptome. Nat. Biotechnol. 31, 1009-1014 (2013).

17. Treutlein, B., Gokce, O., Quake, S. R. \& Sudhof, T. C. Cartography of neurexin alternative splicing mapped by single-molecule long-read mRNA sequencing. Proc. Natl Acad. Sci. USA 111, E1291-E1299 (2014).

18. Bolisetty, M. T., Rajadinakaran, G. \& Graveley, B. R. Determining exon connectivity in complex mRNAs by nanopore sequencing. Genome Biol. 16, 204 (2015).

19. de Jong, L. C. et al. Nanopore sequencing of full-length BRCA1 mRNA transcripts reveals co-occurrence of known exon skipping events. Breast Cancer Res. 19, 127 (2017).

20. Hardwick, S. A. et al. Targeted, high-resolution RNA sequencing of noncoding genomic regions associated with neuropsychiatric functions. Front. Genet. 10, 309 (2019).

21. Tardaguila, M. et al. SQANTI: extensive characterization of long-read transcript sequences for quality control in full-length transcriptome identification and quantification. Genome Res. https://doi.org/10.1101/ gr.222976.117 (2018).

22. Sessegolo, C. et al. Transcriptome profiling of mouse samples using nanopore sequencing of cDNA and RNA molecules. Sci. Rep. 9, 14908 (2019).

23. Anvar, S. Y. et al. Full-length mRNA sequencing uncovers a widespread coupling between transcription initiation and mRNA processing. Genome Biol. 19, 46 (2018).

24. Byrne, A. et al. Nanopore long-read RNAseq reveals widespread transcriptional variation among the surface receptors of individual B cells. Nat. Commun. 8, 16027 (2017).

25. Gupta, I. et al. Single-cell isoform RNA sequencing characterizes isoforms in thousands of cerebellar cells. Nat. Biotechnol. https://doi.org/10.1038/nbt.4259 (2018).

26. Baker, S. C. et al. The external RNA controls consortium: a progress report. Nat. Methods 2, 731-734 (2005).

27. Hardwick, S. A. et al. Spliced synthetic genes as internal controls in RNA sequencing experiments. Nat. Methods 13, 792-798 (2016).

28. Tang, A. D. et al. Full-length transcript characterization of SF3B1 mutation in chronic lymphocytic leukemia reveals downregulation of retained introns. Nat. Commun. 11, 1438 (2020).

29. Pertea, M. et al. CHESS: a new human gene catalog curated from thousands of large-scale RNA sequencing experiments reveals extensive transcriptional noise. Genome Biol. 19, 208 (2018).

30. Ojala, D., Montoya, J. \& Attardi, G. tRNA punctuation model of RNA processing in human mitochondria. Nature 290, 470-474 (1981).

31. Mercer, T. R. et al. The human mitochondrial transcriptome. Cell 146, 645-658 (2011)

32. Hatje, K. et al. The landscape of human mutually exclusive splicing. Mol. Syst. Biol. 13, 959 (2017).

33. Tilgner, $H$. et al. Microfluidic isoform sequencing shows widespread splicing coordination in the human transcriptome. Genome Res. 28, 231-242 (2018).

34. Gooding, C. \& Smith, C. W. Tropomyosin exons as models for alternative splicing. Adv. Exp. Med. Biol. 644, 27-42 (2008).

35. Gooding, C. et al. MBNL1 and PTB cooperate to repress splicing of Tpm1 exon 3. Nucleic Acids Res. 41, 4765-4782 (2013). 
36. Oesterreich, F. C. et al. Splicing of nascent RNA coincides with intron exit from RNA polymerase II. Cell 165, 372-381 (2016).

37. Guo, W. et al. RBM20, a gene for hereditary cardiomyopathy, regulates titin splicing. Nat. Med. 18, 766-773 (2012).

38. Maatz, H. et al. RNA-binding protein RBM20 represses splicing to orchestrate cardiac pre-mRNA processing. J. Clin. Invest. 124, 3419-3430 (2014).

39. Brauch, K. M. et al. Mutations in ribonucleic acid binding protein gene cause familial dilated cardiomyopathy. J. Am. Coll. Cardiol. 54, 930-941 (2009).

40. van den Hoogenhof, M. M. G. et al. RBM20 mutations induce an arrhythmogenic dilated cardiomyopathy related to disturbed calcium handling. Circulation 138, 1330-1342 (2018).

41. Schneider, J. W. et al. Dysregulated ribonucleoprotein granules promote cardiomyopathy in RBM20 gene-edited pigs. Nat. Med. 26, 1788-1800 (2020).

42. Briganti, F. et al. iPSC modelling of RBM20-deficient DCM identifies upregulation of RBM20 as a therapeutic strategy. Cell Rep. 32, 108117(2020).

43. Rego, S. et al. High-frequency actionable pathogenic exome variants in an average-risk cohort. Cold Spring Harb Mol Case Stud 4, a003178 (2018).

44. Love, M. I., Huber, W. \& Anders, S. Moderated estimation of fold change and dispersion for RNA-seq data with DESeq2. Genome Biol. 15, 550 (2014).

45. Pelechano, V., Wei, W. \& Steinmetz, L. M. Extensive transcriptional heterogeneity revealed by isoform profiling. Nature 497, 127-131 (2013).

46. Zhang, Z. et al. Noisy splicing, more than expression regulation, explains why some exons are subject to nonsense-mediated mRNA decay. BMC Biol. 7, 23 (2009).

\section{Acknowledgements}

We thank Lars Velten, Aaron Brooks, Markus Grosch, Benedikt Rauscher, Julia Kornienko, and Sibylle Vonesch for helpful discussion and critical comments on the manuscript. We thank Life Science Editors for their editorial assistance. This study was supported by the National Natural Science Foundation of China (Grant No: 81870187) and National Key R\&D Program of China (2017YFC0908405) to W.W., the Chan Zuckerberg Foundation (2019-202666) to L.M.S., the Steinmetz Cardiomyopathy Fund to L.M.S., and the Walter V. and Idun Berry Fellowship to J.W.

\section{Author contributions}

W.W., C.Z., J.W., and L.M.S. conceived the project. J.W. developed the full-length sequencing method and performed the sequencing experiments. H.S. performed the short-read analysis. F.B. generated the iPSC-CMs. C.Z. established the FulQuant method with inputs from W.W.; C.Z. and W.W. performed the analysis on long-read data. B.M. interpreted RBM20 data on clinical perspectives. C.Z., J.W., W.W., and L.M.S. wrote the manuscript.

\section{Funding}

Open Access funding enabled and organized by Projekt DEAL.

\section{Competing interests}

The authors declare no competing interests.

\section{Additional information}

Supplementary information The online version contains supplementary material available at https://doi.org/10.1038/s41467-021-24484-z.

Correspondence and requests for materials should be addressed to W.W. or L.M.S.

Peer review information Nature Communications thanks Timothy Mercer and the other, anonymous, reviewer(s) for their contribution to the peer review of this work.

Reprints and permission information is available at http://www.nature.com/reprints

Publisher's note Springer Nature remains neutral with regard to jurisdictional claims in published maps and institutional affiliations.

(c) (i) Open Access This article is licensed under a Creative Commons Attribution 4.0 International License, which permits use, sharing, adaptation, distribution and reproduction in any medium or format, as long as you give appropriate credit to the original author(s) and the source, provide a link to the Creative Commons license, and indicate if changes were made. The images or other third party material in this article are included in the article's Creative Commons license, unless indicated otherwise in a credit line to the material. If material is not included in the article's Creative Commons license and your intended use is not permitted by statutory regulation or exceeds the permitted use, you will need to obtain permission directly from the copyright holder. To view a copy of this license, visit http://creativecommons.org/ licenses/by/4.0/.

(C) The Author(s) 2021 